Egyptian Journal of Aquatic Biology \& Fisheries

Zoology Department, Faculty of Science,

Ain Shams University, Cairo, Egypt.

ISSN $1110-6131$

Vol. 25(6): 445 - 466 (2021)

www.ejabf.journals.ekb.eg

\title{
Seasonal variations in the abundance and diversity of zooplankton community inhabiting River Nile and its branches at Qena governorate, Upper Egypt
}

\author{
Wafaa A. Mohammad ${ }^{1 *}$, Ahmad H. Obuid-Allah' ${ }^{2}$, Ahmed S. Moustafa ${ }^{3}$, \\ and Azza M. Gaber ${ }^{3}$ \\ ${ }^{1}$ Zoology Department, Faculty of Science, New Valley University, Egypt \\ ${ }^{2}$ Zoology Department, Faculty of Science, Assiut University, Egypt \\ ${ }^{3}$ Zoology Department, Faculty of Science, South Valley University, Qena, Egypt \\ *Corresponding Author: Wafaa.moh@sci.nvu.edu.eg
}

\section{ARTICLE INFO}

Article History:

Received: Dec. 11, 2021

Accepted: Dec. 21, 2021

Online: Dec. 30, 2021

Keywords:

Cladocera,

Copepoda,

Ostracoda,

Rotifera,

Composition,

Physicochemical

measurements

\section{ABSTRACT}

In the present study, the diversity and seasonal variation of the freshwater zooplankton were studied throughout a period of one year (September 2020 to August 2021). Sampling collections were monthly carried out from fifteen sites representing 7 cities located north, and south to Qena city from the River Nile and its branches $\left(26^{\circ} 9^{\prime} 18.22^{\prime \prime} \mathrm{N}\right.$ and a longitude of 32 42'57.64"E). Physiochemical factors of water of the different collecting sites were measured and analysed using One-way Analysis of variance (ANOVA), and 195056 individuals were collected. Shannon-Weiner diversity, Equitability and Margalef's indices were used to determine zooplankton composition. The results revealed that there were 48 taxonomic units dominated by cladocerans $(87 \%, 23$ taxa) followed by copepods $(11.74 \%, 12$ taxa), rotifers $(1.01 \%, 8$ taxa), and ostracods $(0.24 \%, 3$ taxa). The most common species of Cladocera was Chydorus sphaericus representing $62.95 \%$; while the most common of Copepoda was Messocyclops ogunnus representing 34.71\%. The most common species of Rotifera was Lecane lune representing $34.80 \%$; whereas, the most common of Ostracoda was Cypridopsis vidua representing $96.22 \%$. Both zooplankton communities and populations of Cladocera showed higher density during the rainy season. Investigation on the physiochemical parameters showed that $\mathrm{pH}$, turbidity, and dissolved oxygen all recorded maximum values in the autumn season. Furthermore, both the total dissolved solids and conductivity recorded maximum values in the winter season. Total abundance of zooplankton showed a positive relationship with Taxa richness, ShannonWeiner diversity, and Margalef's index and Equitability. They showed their peaks during the winter season, while the evenness index increased in the dry season.

\section{INTRODUCTION}

Zooplankton group is naturally cosmopolitan and plays a vital role in aquatic ecosystems. They can be found in a variety of freshwater habitats, including contaminated, industrial, and municipal wastewaters (Savitha \& Yamakanamardi, 2012). In freshwater bodies, the zooplankton community constitutes an extremely diverse 
assemblage of organisms represented by most of the invertebrate phyla. Generally, zooplanktons are one of the most essential biotic components, influencing all functional elements such as food chains, energy flow, and matter cycling (Trivedi et al., 2015; Gupta et al., 2016; González et al., 2018). They are strong bio-indicators of physical and chemical parameters of water, hence playing an important role in the evaluation of the trophic status of water (Berraho et al., 2019; Okechukwu, 2019). This is due to the fact that they frequently display dramatic changes in the response to changes in the biotic and physicochemical features of the aquatic environment. Naturally, Zooplanktons are extremely susceptible to environmental changes, nutrient enrichment, and various levels of pollution, so, plankton communities fluctuate in terms of tolerance, abundance, dominancy, and diversity in their habitat (Madhusudhana et al., 2014).

Species diversity mainly refers to the number of different species in the community including both abundant and uncommon species. Moreover, it is very high in natural communities like tropical and subtropical, while it is very low in physically or human-controlled communities. Zooplankton diversity acts as an important indicator to detect pollution, water quality, and eutrophication in freshwater ecosystems (Güher, 2014). The diversity and abundance of zooplankton species are critical indicators of a location's potential fisheries resources (Varadharajan et al., 2009). Diversity indices have been used as an important tool by ecologists to understand community structure in terms of richness, Evenness, or the total number of existing individuals (Wilhm \& Dorris, 1968; Allan, 1975).

Variations in both physicochemical properties, as well as biotic factors such as feeding ecology and predator pressure, have a significant impact on the distribution and diversity of zooplankton (Egborge, 1994). Previous studies indicated that physicochemical conditions of the aquatic environments can cause changes in the qualitative and quantitative composition of zooplankton and influence their densities (Lin et al., 2003; Rita et al., 2005 and Obuid-allah et al., 2019, 2020). Nevertheless, not much is known on all the factors that control the seasonal variations of zooplankton abundance and diversity in freshwater ecosystems thus, it should be a concern to new and intending researchers for further studies. The aim of the present study was to estimate seasonal variation in zooplankton abundance and diversity in the River Nile and its branches at Qena Governorate.

\section{MATERIALS AND METHODS}

\section{Study areas}

In the present study 15 sites representing 7 cities were selected lying between

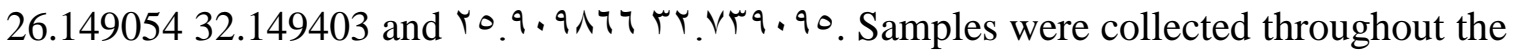
period of study (from September 2020 to August 2021). The samples were collected from 15 sites ( 7 sites from the main course of the Nile stream (from site 1 to site 7) and 8 sites 
from its tributaries (from site 8 to site 15). sites of collections were identified and marked using a geographical positioning system (GPS) Fig. (1).

2. Water quality parameters:

Different physicochemical factors were measured monthly using electronic portable instruments such as $\mathrm{pH}$, total dissolved solids (TDS), conductivity, dissolved oxygen.

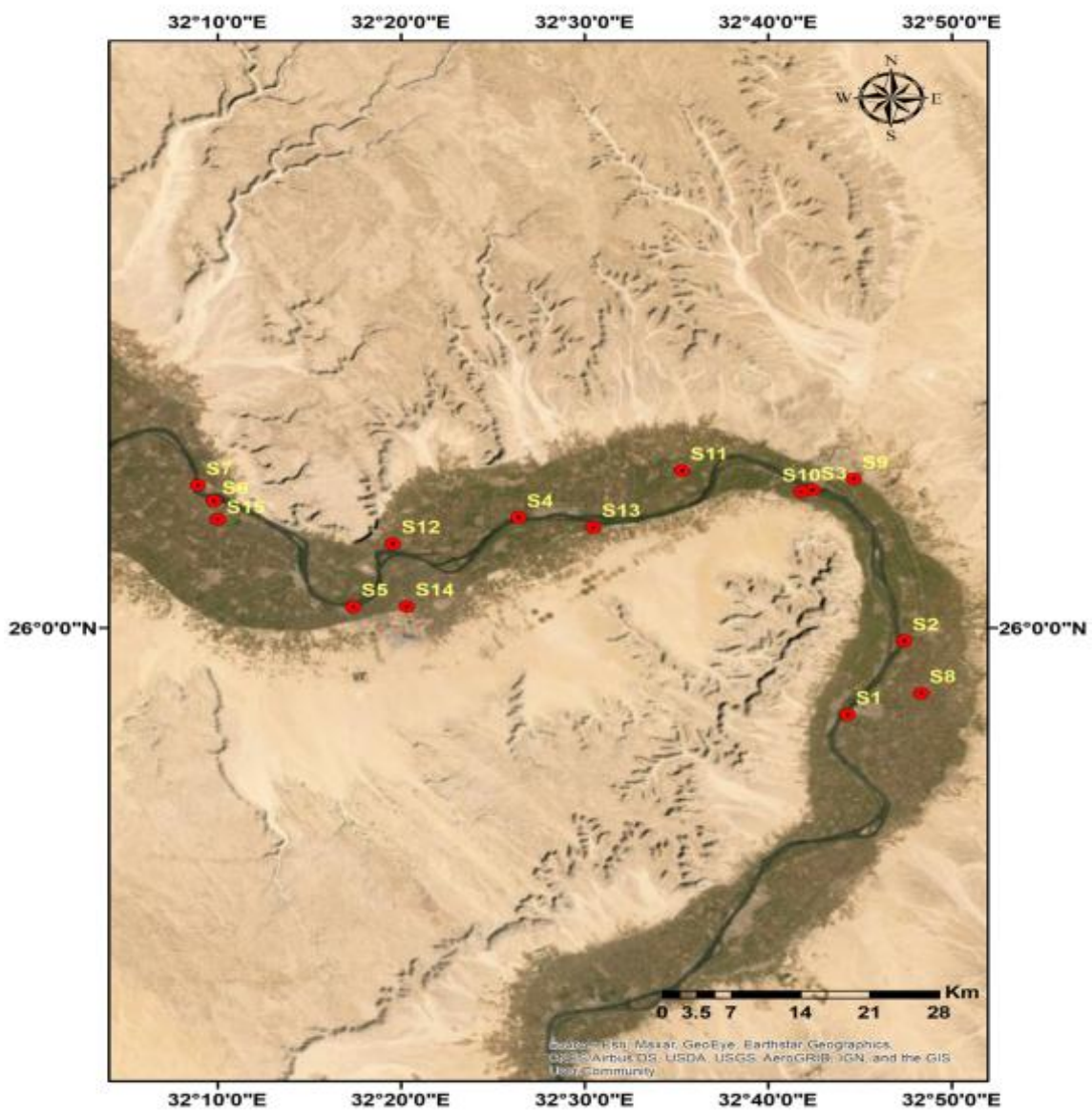

Fig. 1. Map showing the locations of studied sites.

\section{Zooplankton sampling:}

Zooplankton samples were collected monthly using towing plankton net $(12.7 \mathrm{~cm}$ diameter, $38 \mathrm{~cm}$ in length and $153 \mu \mathrm{m}$ mesh size). The collected individuals were preserved in $95 \%$ ethanol and allowed to settle in $24 \mathrm{~h}$. Identification was carried out using relevant keys including (Brooks, 1959; Wilson \& Yeatman, 1959; Obuid-Allah, 2001; Elfeky \& Sayed, 2014). Samples were examined under an OPTIKA microscope. 


\section{Statistical analysis:}

One-way Analysis of variance (ANOVA) was used to determine the mean monthly variation of zooplankton and physicochemical parameters of the investigated sites. To measure the stability of zooplankton structure, diversity indices: species richness (Margalef, 1968), Shannon-Wiener diversity index (Shannon and Wiener, 1963), evenness and equitability (Pielou, 1975) were calculated by using (past v3. 22). Principal component analysis (PCA) was performed by using (origin pro-2021), to show the similarity between the investigated sites in diversity indices including, Taxa_S, Margalef, Shannon_H, Equitability and Evenness. A Canonical Correspondence Analysis (CCA), was used to determine the most significant physicochemical variables affecting the biotic community by intuitively elucidating the relationship between environmental factors and zooplankton groups. CCA analysis was performed using abundance data of zooplankton groups that were $(0.1 \%$ of the total zooplankton). The environmental variables were selected for this analysis including $\mathrm{pH}$, total dissolved solids (TDS), conductivity, dissolved oxygen. The CCA was performed using the CANOCO 4.5 program for Windows system (ter Braak \& $\mathbf{S}^{\swarrow}$ milauer, 2002). In order to identify the similarity between all investigated sites in relation to the abundance of zooplankton, cluster analysis was applied using (origin pro-2021). The relationships between sites and dominancy of zooplankton species were studied by drawing heatmaps by using (origin pro-2021).

\section{RESULTS}

During the sampling period, forty-eight (48) different zooplankton species were encountered (Table 1). Samples were collected from 15 sites located at Qena Governorate, including the River Nile and its branches. The collected zooplankton species have been divided into 4 groups, Cladocera was the most abundant group (23 species), followed by Copepoda (12 species with additionally copepodite stage and Nauplius stage). However, Rotifera was the third dominant one ( 8 species) and the least abundant one was Ostracoda (3 species).

The total number $195056 \mathrm{indv} / \mathrm{m}^{3}$ of different taxa of zooplankton were recorded during the period of study (Table 2). The maximum abundance of Cladocera was recorded at site $13\left(24020 \mathrm{indv} / \mathrm{m}^{3}\right)$, while the minimum abundance was at site $12(1282$ indv $/ \mathrm{m}^{3}$ ). The most abundant species of Cladocera at all investigated sites was Chydorus 
sphaericus $\left(106834 \mathrm{indv} / \mathrm{m}^{3}\right)$. The maximum abundance of Copepoda was noticed at site $13\left(4596 \mathrm{indv} / \mathrm{m}^{3}\right)$, whereas it was recorded $\left(182 \mathrm{indv} / \mathrm{m}^{3}\right)$ at site 12 . The most abundant species of Copepoda was Mesocyclops ogunnus (7947 indv $\mathrm{Im}^{3}$ ). Rotifera recorded the maximum abundance at site $13\left(1110 \mathrm{indv} / \mathrm{m}^{3}\right)$ and no record of its presence was noticed at site 4. The most abundant species of Rotifera at all investigated sites was Lecane lune $\left(688 \mathrm{indv} / \mathrm{m}^{3}\right)$. The highest abundance of Ostracoda was recorded at site $4\left(113 \mathrm{indv} / \mathrm{m}^{3}\right)$ with no evidence of existence at sites 1,8,9,11, and 14. Cypridopsis vidua was the most abundant species of Ostracoda.

Fig. (2) shows that Cladocera constituting $87 \%$ of the total abundance of zooplankton followed by Copepoda $11.74 \%$, Rotifera $1.01 \%$ and Ostracoda $0.24 \%$.

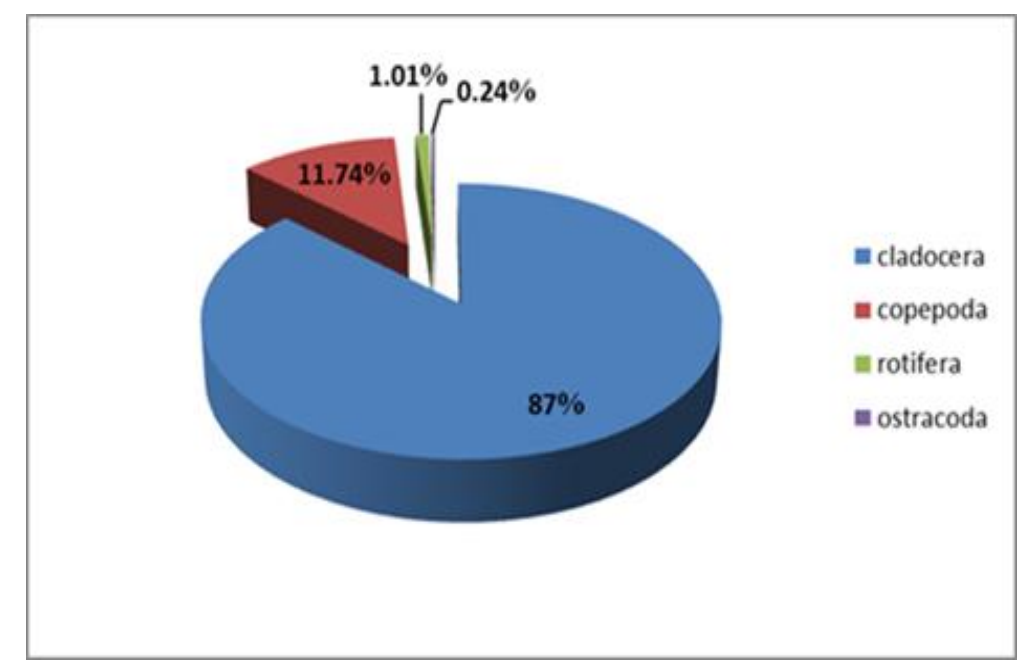

Fig. 2. Percentage contribution of zooplankton taxa recorded in all investigated sites. 
Table 1. Frequancy of zooplankton recorded in all investigated site.

\begin{tabular}{|c|c|c|c|c|c|c|c|c|c|c|c|c|c|c|c|}
\hline Taxa & 氙 & $\stackrel{N}{\stackrel{n}{n}}$ & $\begin{array}{l}\text { m } \\
\stackrel{n}{n}\end{array}$ & 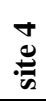 & $\begin{array}{l}n \\
\stackrel{0}{n} \\
0\end{array}$ & 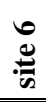 & 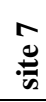 & $\begin{array}{l}\infty \\
\stackrel{y}{n}\end{array}$ & $\stackrel{a}{0}$ & 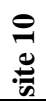 & $=$ & $\frac{N}{\frac{N}{n}}$ & $\frac{m}{\tilde{g}}$ & $\frac{ \pm}{\stackrel{ \pm}{\sigma}}$ & $\frac{n}{5}$ \\
\hline \multicolumn{16}{|l|}{ Cladocera } \\
\hline Bosmina longirostris & + & + & + & + & + & + & + & + & + & + & + & + & + & + & + \\
\hline Simocephalus expinosus & + & + & + & + & + & + & + & + & - & - & - & - & - & + & + \\
\hline Simocephalus vetulus & + & + & + & + & + & + & + & - & + & - & - & - & - & + & + \\
\hline Ceriodaphnia reticulate & + & + & + & + & + & + & + & + & + & + & + & + & + & + & + \\
\hline Daphnia longispina & + & + & - & + & - & + & + & + & - & - & - & - & + & - & + \\
\hline Ilyocryptus sordidus & + & + & + & + & + & + & + & + & + & + & + & + & + & + & + \\
\hline Macrothrix laticornis & + & + & + & + & + & + & + & + & + & + & + & + & + & + & + \\
\hline Alona bukobensis (a) & + & + & + & + & + & - & + & + & + & + & - & - & - & + & - \\
\hline Alona bukobensis (b) & + & + & + & + & + & + & + & + & + & - & + & - & + & + & + \\
\hline Alona bukobensis (c) & + & + & + & + & + & + & + & + & + & + & + & + & + & + & + \\
\hline Alona rectangular & - & - & - & - & - & - & - & + & - & - & - & - & - & - & - \\
\hline Alona sp. & - & - & - & - & + & - & - & - & - & - & - & - & - & - & - \\
\hline Euryalona sp. & - & - & - & - & + & - & - & - & - & - & - & - & - & - & - \\
\hline Camptocercus australis & - & + & + & - & - & - & - & + & - & - & - & - & - & - & - \\
\hline Leydigia quadrangularis & - & - & - & - & + & - & - & - & - & - & - & - & - & - & - \\
\hline Chydorus sphaericus & + & + & + & + & + & + & + & + & + & + & + & + & + & + & + \\
\hline Chydorus sp. & - & - & + & - & - & - & - & - & - & - & - & - & - & - & - \\
\hline Disparalona rostrata. & + & + & + & + & + & + & + & + & + & + & - & - & + & + & + \\
\hline Pleuroxus straminius & - & - & - & - & - & + & - & - & - & - & - & - & - & - & - \\
\hline Pleuroxus aduncus & - & + & - & - & - & - & - & - & - & - & - & - & - & - & - \\
\hline Dunhevedia crassa & - & + & - & - & + & - & - & - & - & - & - & - & - & - & - \\
\hline Diaphanosoma birgei & - & + & - & - & - & - & + & - & - & - & - & - & - & + & - \\
\hline Moina micrura & + & + & + & - & - & - & + & - & - & - & + & - & - & - & - \\
\hline \multicolumn{16}{|l|}{ Copepoda } \\
\hline Thermodiaptomus galebi & + & + & + & + & + & + & + & + & + & + & + & + & - & + & + \\
\hline Mesocyclops ogunnus & + & + & + & + & + & + & + & + & + & + & + & - & + & + & + \\
\hline Thermocyclops consimilis & + & + & + & + & + & + & + & + & + & + & + & - & + & + & + \\
\hline Thermocyclops neglectus & + & + & + & + & + & - & - & - & - & + & + & - & - & - & - \\
\hline Tropocyclops confinis & + & - & + & + & + & - & + & - & + & + & - & - & - & + & + \\
\hline Macrocyclops albidus & - & + & - & - & + & - & + & + & - & - & - & - & - & - & - \\
\hline Microcylops varicans & + & + & + & + & + & + & + & + & + & + & + & - & + & + & + \\
\hline Microcylops linjanticus & + & + & + & + & + & + & + & - & + & + & - & - & + & + & + \\
\hline Paracyclops fimbriatus & - & - & - & - & - & - & - & - & - & + & - & - & - & - & - \\
\hline Afrocyclops gibsoni & - & + & - & - & + & - & - & - & - & + & - & - & - & - & - \\
\hline Eucyclops serrulatus & - & - & - & - & - & - & + & - & - & - & - & - & + & - & - \\
\hline Shizopera nilotica & - & + & - & + & + & + & + & + & + & + & + & + & + & + & - \\
\hline Copepodite stage & + & + & + & + & + & + & + & + & + & + & + & + & + & + & + \\
\hline Nauplius stage & + & - & - & - & + & - & + & - & - & - & + & + & - & - & - \\
\hline \multicolumn{16}{|l|}{$\begin{array}{l}\text { Rotifera } \\
\text { R }\end{array}$} \\
\hline Cephalodella gibba & + & - & - & - & + & - & + & + & + & - & + & + & + & + & + \\
\hline Lecane lune & + & + & + & - & + & - & + & + & + & - & + & - & + & - & + \\
\hline Brachionus rubens & + & - & - & - & + & - & - & - & - & - & - & - & + & - & + \\
\hline Brachionus qudridentatus & - & - & - & - & - & - & - & - & - & + & - & - & - & + & - \\
\hline Brachionus zahniseri & - & + & - & - & - & + & - & - & - & + & - & - & - & - & - \\
\hline Brachionus sp. & - & - & + & - & - & - & - & - & - & - & - & - & - & - & - \\
\hline Brachionus urceolaris & - & - & - & - & - & - & - & - & - & - & - & - & - & - & + \\
\hline Trichocerca porcellus & + & - & - & - & - & - & + & - & - & - & - & - & - & - & - \\
\hline \multicolumn{16}{|l|}{ Ostracoda } \\
\hline Cypridopsis vidua & - & + & + & + & + & + & + & - & - & + & - & + & + & - & + \\
\hline Potamocypris variegate & - & - & - & - & - & - & - & - & - & + & - & - & - & - & - \\
\hline Candona sp. & - & + & - & - & - & - & - & - & - & - & - & - & - & - & - \\
\hline
\end{tabular}

(+ present , - absent) 
Table 2. Density and relative abundance of zooplankton species in all investigated sites.

\begin{tabular}{|c|c|c|c|c|c|c|c|c|c|c|c|c|c|c|}
\hline Taxa & $\stackrel{\mathscr{C}}{\overparen{\theta}}$ & $\partial^{\circ}$ & $\stackrel{\mathscr{C}}{\overparen{O}}$ & $a^{\circ}$ & ڤ્ఝ & $\partial^{\circ}$ & 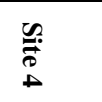 & $\partial^{\circ}$ & 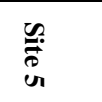 & $0^{\circ}$ & 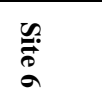 & $\partial^{\circ}$ & $\stackrel{\mathscr{O}}{\widetilde{\sigma}}$ & $a^{\circ}$ \\
\hline Cladocera & 14135 & 7.247 & 13017 & 6.673 & 10283 & 5.272 & 10131 & 5.194 & 14222 & 7.291 & 13046 & 6.688 & 14567 & 7.468 \\
\hline Bosmina longirostris & 1155 & 0.592 & 845 & 0.433 & 91 & 0.047 & 238 & 0.122 & 297 & 0.152 & 155 & 0.079 & 185 & 0.095 \\
\hline Simocephalus expinosus & 69 & 0.035 & 70 & 0.036 & 20 & 0.010 & 13 & 0.007 & 187 & 0.096 & 120 & 0.062 & 46 & 0.024 \\
\hline Simocephalus vetulus & 18 & 0.009 & 11 & 0.006 & 7 & 0.004 & 13 & 0.007 & 24 & 0.012 & 71 & 0.036 & 7 & 0.004 \\
\hline Ceriodaphnia reticulata & 2516 & 1.290 & 1991 & 1.021 & 1186 & 0.608 & 3011 & 1.544 & 1874 & 0.961 & 2904 & 1.489 & 1576 & 0.808 \\
\hline Daphnia longispina & 27 & 0.014 & 24 & 0.012 & 0 & 0.000 & 23 & 0.012 & 0 & 0.000 & 20 & 0.010 & 25 & 0.013 \\
\hline Ilyocryptus sordidus & 181 & 0.093 & 235 & 0.120 & 232 & 0.119 & 223 & 0.114 & 368 & 0.189 & 146 & 0.075 & 473 & 0.242 \\
\hline Macrothrix laticornis & 245 & 0.126 & 469 & 0.240 & 59 & 0.030 & 40 & 0.021 & 62 & 0.032 & 27 & 0.014 & 282 & 0.145 \\
\hline Alona bukobensis (a) & 5 & 0.003 & 13 & 0.007 & 60 & 0.031 & 213 & 0.109 & 7 & 0.004 & 0 & 0.000 & 90 & 0.046 \\
\hline Alona bukobensis ( $b$ ) & 215 & 0.110 & 132 & 0.068 & 39 & 0.020 & 353 & 0.181 & 733 & 0.376 & 556 & 0.285 & 418 & 0.214 \\
\hline Alona bukobensis (c) & 98 & 0.050 & 65 & 0.033 & 382 & 0.196 & 465 & 0.238 & 145 & 0.074 & 72 & 0.037 & 356 & 0.183 \\
\hline Alona rectangular & 0 & 0.000 & 0 & 0.000 & 0 & 0.000 & 0 & 0.000 & 0 & 0.000 & 0 & 0.000 & 0 & 0.000 \\
\hline Alona sp. & 0 & 0.000 & 0 & 0.000 & 0 & 0.000 & 0 & 0.000 & 13 & 0.007 & 0 & 0.000 & 0 & 0.000 \\
\hline Euryalona sp. & 0 & 0.000 & 0 & 0.000 & 0 & 0.000 & 0 & 0.000 & 80 & 0.041 & 0 & 0.000 & 0 & 0.000 \\
\hline Camptocercus australis & 0 & 0.000 & 13 & 0.007 & 11 & 0.006 & 0 & 0.000 & 0 & 0.000 & 0 & 0.000 & 0 & 0.000 \\
\hline Leydigia quadrangularis & 0 & 0.000 & 0 & 0.000 & 0 & 0.000 & 0 & 0.000 & 33 & 0.017 & 0 & 0.000 & 0 & 0.000 \\
\hline Chydorus sphaericus & 8673 & 4.446 & 8345 & 4.278 & 7604 & 3.898 & 5285 & 2.709 & 9002 & 4.615 & 7807 & 4.002 & 10662 & 5.466 \\
\hline Chydorus sp. & 0 & 0.000 & 0 & 0.000 & 13 & 0.007 & 0 & 0.000 & 0 & 0.000 & 0 & 0.000 & 0 & 0.000 \\
\hline Disparalona rostrata. & 867 & 0.444 & 742 & 0.380 & 566 & 0.290 & 254 & 0.130 & 1370 & 0.702 & 1161 & 0.595 & 295 & 0.151 \\
\hline Pleuroxus straminius & 0 & 0.000 & 0 & 0.000 & 0 & 0.000 & 0 & 0.000 & 0 & 0.000 & 7 & 0.004 & 0 & 0.000 \\
\hline Pleuroxus aduncus & 0 & 0.000 & 13 & 0.007 & 0 & 0.000 & 0 & 0.000 & 0 & 0.000 & 0 & 0.000 & 0 & 0.000 \\
\hline Dunhevedia crassa & 0 & 0.000 & 18 & 0.009 & 0 & 0.000 & 0 & 0.000 & 27 & 0.014 & 0 & 0.000 & 0 & 0.000 \\
\hline Diaphanosoma birgei & 0 & 0.000 & 4 & 0.002 & 0 & 0.000 & 0 & 0.000 & 0 & 0.000 & 0 & 0.000 & 13 & 0.007 \\
\hline Moina micrura & 66 & 0.034 & 27 & 0.014 & 13 & 0.007 & 0 & 0.000 & 0 & 0.000 & 0 & 0.000 & 139 & 0.071 \\
\hline Copepoda & 1574 & 0.807 & 1236 & 0.634 & 864 & 0.443 & 1599 & 0.820 & 1488 & 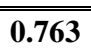 & 1102 & 0.565 & 1459 & 0.748 \\
\hline Thermodiaptomus galebi & 407 & 0.209 & 561 & 0.288 & 24 & 0.012 & 459 & 0.235 & 114 & 0.058 & 175 & 0.090 & 65 & 0.033 \\
\hline Mesocyclops ogunnus & 406 & 0.208 & 276 & 0.141 & 503 & 0.258 & 472 & 0.242 & 781 & 0.400 & 637 & 0.327 & 680 & 0.349 \\
\hline Thermocyclops consimilis & 378 & 0.194 & 162 & 0.083 & 122 & 0.063 & 336 & 0.172 & 339 & 0.174 & 169 & 0.087 & 134 & 0.069 \\
\hline Thermocyclops neglectus & 52 & 0.027 & 22 & 0.011 & 13 & 0.007 & 60 & 0.031 & 17 & 0.009 & 0 & 0.000 & 0 & 0.000 \\
\hline Tropocyclops confinis & 26 & 0.013 & 0 & 0.000 & 21 & 0.011 & 41 & 0.021 & 13 & 0.007 & 0 & 0.000 & 26 & 0.013 \\
\hline Macrocyclops albidus & 0 & 0.000 & 7 & 0.004 & 0 & 0.000 & 0 & 0.000 & 7 & 0.004 & 0 & 0.000 & 26 & 0.013 \\
\hline Microcylops varicans & 70 & 0.036 & 44 & 0.023 & 20 & 0.010 & 26 & 0.013 & 30 & 0.015 & 25 & 0.013 & 155 & 0.079 \\
\hline Microcylops linjanticus & 98 & 0.050 & 72 & 0.037 & 59 & 0.030 & 48 & 0.025 & 45 & 0.023 & 81 & 0.042 & 202 & 0.104 \\
\hline Paracyclops fimbriatus & 0 & 0.000 & 0 & 0.000 & 0 & 0.000 & 0 & 0.000 & 0 & 0.000 & 0 & 0.000 & 0 & 0.000 \\
\hline Afrocyclops gibsoni & 0 & 0.000 & 7 & 0.004 & 0 & 0.000 & 0 & 0.000 & 27 & 0.014 & 0 & 0.000 & 0 & 0.000 \\
\hline Eucyclops serrulatus & 0 & 0.000 & 0 & 0.000 & 0 & 0.000 & 0 & 0.000 & 0 & 0.000 & 0 & 0.000 & 13 & 0.007 \\
\hline Shizopera nilotica & 0 & 0.000 & 7 & 0.004 & 0 & 0.000 & 52 & 0.027 & 29 & 0.015 & 11 & 0.006 & 20 & 0.010 \\
\hline Copepodite stage & 124 & 0.064 & 78 & 0.040 & 102 & 0.052 & 105 & 0.054 & 73 & 0.037 & 4 & 0.002 & 81 & 0.042 \\
\hline Nauplius stage & 13 & 0.007 & 0 & 0.000 & 0 & 0.000 & 0 & 0.000 & 13 & 0.007 & 0 & 0.000 & 57 & 0.029 \\
\hline Rotifera & 115 & 0.059 & 26 & 0.013 & 11 & $\overline{0.006}$ & 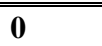 & 0.000 & 53 & 0.027 & 7 & 0.004 & 58 & $\overline{0.030}$ \\
\hline Cephalodella gibba & 5 & 0.003 & 0 & 0.000 & 0 & 0.000 & 0 & 0.000 & 13 & 0.007 & 0 & 0.000 & 13 & 0.007 \\
\hline Lecane lune & 79 & 0.041 & 13 & 0.007 & 4 & 0.002 & 0 & 0.000 & 13 & 0.007 & 0 & 0.000 & 32 & 0.016 \\
\hline Brachionus rubens & 26 & 0.013 & 0 & 0.000 & 0 & 0.000 & 0 & 0.000 & 27 & 0.014 & 0 & 0.000 & 0 & 0.000 \\
\hline Brachionus qudridentatus & 0 & 0.000 & 0 & 0.000 & 0 & 0.000 & 0 & 0.000 & 0 & 0.000 & 0 & 0.000 & 0 & 0.000 \\
\hline Brachionus zahniseri & 0 & 0.000 & 13 & 0.007 & 0 & 0.000 & 0 & 0.000 & 0 & 0.000 & 7 & 0.004 & 0 & 0.000 \\
\hline Brachionus sp. & 0 & 0.000 & 0 & 0.000 & 7 & 0.004 & 0 & 0.000 & 0 & 0.000 & 0 & 0.000 & 0 & 0.000 \\
\hline Brachionus urceolaris & 0 & 0.000 & 0 & 0.000 & 0 & 0.000 & 0 & 0.000 & 0 & 0.000 & 0 & 0.000 & 0 & 0.000 \\
\hline Trichocerca porcellus & 5 & 0.003 & 0 & 0.000 & 0 & 0.000 & 0 & 0.000 & 0 & 0.000 & 0 & 0.000 & 13 & 0.007 \\
\hline Ostracoda & 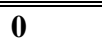 & $\overline{0.000}$ & 9 & 0.005 & 9 & $\overline{0.005}$ & 113 & 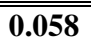 & 24 & $\overline{0.012}$ & 18 & 0.009 & 49 & 0.025 \\
\hline Cypridopsis vidua & 0 & 0.000 & 4 & 0.002 & 9 & 0.005 & 113 & 0.058 & 24 & 0.012 & 18 & 0.009 & 49 & 0.025 \\
\hline Potamocypris variegate & 0 & 0.000 & 0 & 0.000 & 0 & 0.000 & 0 & 0.000 & 0 & 0.000 & 0 & 0.000 & 0 & 0.000 \\
\hline Candona sp. & 0 & 0.000 & 5 & 0.003 & 0 & 0.000 & 0 & 0.000 & 0 & 0.000 & 0 & 0.000 & 0 & 0.000 \\
\hline
\end{tabular}


Table 2. Contained

\begin{tabular}{|c|c|c|c|c|c|c|c|c|c|c|c|c|c|c|c|c|}
\hline Taxa & 竞 & $0^{\circ}$ & 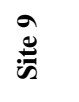 & $a^{\circ}$ & 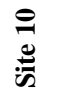 & $0^{\circ}$ & $\begin{array}{l}\beth \\
\stackrel{\Xi}{*}\end{array}$ & $a^{\circ}$ & 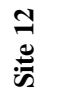 & $a^{\circ}$ & $\stackrel{2}{\stackrel{2}{*}}$ & $\partial^{\circ}$ & $\frac{\Delta}{\stackrel{\Xi}{a}}$ & $\partial^{\circ}$ & $\frac{n}{\stackrel{n}{*}}$ & $a^{\circ}$ \\
\hline Cladocera & 7280 & 3.732 & 6448 & 3.306 & 9462 & 4.851 & 4320 & 2.215 & 1282 & 0.657 & 24020 & 12.314 & 15727 & 8.063 & 11766 & 6.032 \\
\hline Bosmina longirostris & 1237 & 0.634 & 294 & 0.151 & 87 & 0.045 & 164 & 0.084 & 344 & 0.176 & 208 & 0.107 & 291 & 0.149 & 411 & 0.211 \\
\hline Simocephalus expinosus & 26 & 0.013 & 0 & 0.000 & 0 & 0.000 & 0 & 0.000 & 0 & 0.000 & 0 & 0.000 & 67 & 0.034 & 73 & 0.037 \\
\hline Simocephalus vetulus & 0 & 0.000 & 44 & 0.023 & 0 & 0.000 & 0 & 0.000 & 0 & 0.000 & 0 & 0.000 & 13 & 0.007 & 33 & 0.017 \\
\hline Ceriodaphnia reticulata & 1413 & 0.724 & 2061 & 1.057 & 509 & 0.261 & 725 & 0.372 & 73 & 0.037 & 3217 & 1.649 & 4401 & 2.256 & 1131 & 0.580 \\
\hline Daphnia longispina & 9 & 0.005 & 0 & 0.000 & 0 & 0.000 & 0 & 0.000 & 0 & 0.000 & 51 & 0.026 & 0 & 0.000 & 209 & 0.107 \\
\hline Ilyocryptus sordidus & 144 & 0.074 & 310 & 0.159 & 86 & 0.044 & 147 & 0.075 & 212 & 0.109 & 1814 & 0.930 & 266 & 0.136 & 150 & 0.077 \\
\hline Macrothrix laticornis & 319 & 0.164 & 18 & 0.009 & 35 & 0.018 & 119 & 0.061 & 173 & 0.089 & 1342 & 0.688 & 400 & 0.205 & 89 & 0.046 \\
\hline Alona bukobensis (a) & 256 & 0.131 & 13 & 0.007 & 184 & 0.094 & 0 & 0.000 & 0 & 0.000 & 0 & 0.000 & 69 & 0.035 & 0 & 0.000 \\
\hline Alona bukobensis (b) & 145 & 0.074 & 54 & 0.028 & 0 & 0.000 & 26 & 0.013 & 0 & 0.000 & 424 & 0.217 & 152 & 0.078 & 552 & 0.283 \\
\hline Alona bukobensis (c) & 257 & 0.132 & 24 & 0.012 & 1283 & 0.658 & 70 & 0.036 & 137 & 0.070 & 1312 & 0.673 & 650 & 0.333 & 53 & 0.027 \\
\hline Alona rectangular & 26 & 0.013 & 0 & 0.000 & 0 & 0.000 & 0 & 0.000 & 0 & 0.000 & 0 & 0.000 & 0 & 0.000 & 0 & 0.000 \\
\hline Alona sp. & 0 & 0.000 & 0 & 0.000 & 0 & 0.000 & 0 & 0.000 & 0 & 0.000 & 0 & 0.000 & 0 & 0.000 & 0 & 0.000 \\
\hline Euryalona sp. & 0 & 0.000 & 0 & 0.000 & 0 & 0.000 & 0 & 0.000 & 0 & 0.000 & 0 & 0.000 & 0 & 0.000 & 0 & 0.000 \\
\hline Camptocercus australis & 26 & 0.013 & 0 & 0.000 & 0 & 0.000 & 0 & 0.000 & 0 & 0.000 & 0 & 0.000 & 0 & 0.000 & 0 & 0.000 \\
\hline Leydigia quadrangularis & 0 & 0.000 & 0 & 0.000 & 0 & 0.000 & 0 & 0.000 & 0 & 0.000 & 0 & 0.000 & 0 & 0.000 & 0 & 0.000 \\
\hline Chydorus sphaericus & 3237 & 1.660 & 3412 & 1.749 & 7252 & 3.718 & 3056 & 1.567 & 343 & 0.176 & 14916 & 7.647 & 9178 & 4.705 & 8062 & 4.133 \\
\hline Chydorus sp. & 0 & 0.000 & 0 & 0.000 & 0 & 0.000 & 0 & 0.000 & 0 & 0.000 & 0 & 0.000 & 0 & 0.000 & 0 & 0.000 \\
\hline Disparalona rostrata. & 185 & 0.095 & 218 & 0.112 & 26 & 0.013 & 0 & 0.000 & 0 & 0.000 & 736 & 0.377 & 64 & 0.033 & 1003 & 0.514 \\
\hline Pleuroxus straminius & 0 & 0.000 & 0 & 0.000 & 0 & 0.000 & 0 & 0.000 & 0 & 0.000 & 0 & 0.000 & 0 & 0.000 & 0 & 0.000 \\
\hline Pleuroxus aduncus & 0 & 0.000 & 0 & 0.000 & 0 & 0.000 & 0 & 0.000 & 0 & 0.000 & 0 & 0.000 & 0 & 0.000 & 0 & 0.000 \\
\hline Dunhevedia crassa & 0 & 0.000 & 0 & 0.000 & 0 & 0.000 & 0 & 0.000 & 0 & 0.000 & 0 & 0.000 & 0 & 0.000 & 0 & 0.000 \\
\hline Diaphanosoma birgei & 0 & 0.000 & 0 & 0.000 & 0 & 0.000 & 0 & 0.000 & 0 & 0.000 & 0 & 0.000 & 176 & 0.090 & 0 & 0.000 \\
\hline Moina micrura & 0 & 0.000 & 0 & 0.000 & 0 & 0.000 & 13 & 0.007 & 0 & 0.000 & 0 & 0.000 & 0 & 0.000 & 0 & 0.000 \\
\hline Copepoda & 631 & 0.323 & 1095 & 0.561 & 3225 & 1.653 & 774 & 0.397 & 182 & 0.093 & 4596 & 2.356 & 2133 & 1.094 & 939 & 0.481 \\
\hline Thermodiaptomus galebi & 57 & 0.029 & 193 & 0.099 & 202 & 0.104 & 131 & 0.067 & 26 & 0.013 & 0 & 0.000 & 120 & 0.062 & 137 & 0.070 \\
\hline Mesocyclops ogunnus & 293 & 0.150 & 294 & 0.151 & 1198 & 0.614 & 366 & 0.188 & 0 & 0.000 & 424 & 0.217 & 1084 & 0.556 & 533 & 0.273 \\
\hline Thermocyclops consimilis & 86 & 0.044 & 254 & 0.130 & 508 & 0.260 & 78 & 0.040 & 0 & 0.000 & 125 & 0.064 & 519 & 0.266 & 184 & 0.094 \\
\hline Thermocyclops neglectus & 0 & 0.000 & 0 & 0.000 & 39 & 0.020 & 26 & 0.013 & 0 & 0.000 & 0 & 0.000 & 0 & 0.000 & 0 & 0.000 \\
\hline Tropocyclops confinis & 0 & 0.000 & 56 & 0.029 & 61 & 0.031 & 0 & 0.000 & 0 & 0.000 & 0 & 0.000 & 13 & 0.007 & 13 & 0.007 \\
\hline Macrocyclops albidus & 13 & 0.007 & 0 & 0.000 & 0 & 0.000 & 0 & 0.000 & 0 & 0.000 & 0 & 0.000 & 0 & 0.000 & 0 & 0.000 \\
\hline Microcylops varicans & 18 & 0.009 & 13 & 0.007 & 117 & 0.060 & 65 & 0.033 & 0 & 0.000 & 56 & 0.029 & 13 & 0.007 & 33 & 0.017 \\
\hline Microcylops linjanticus & 0 & 0.000 & 160 & 0.082 & 332 & 0.170 & 0 & 0.000 & 0 & 0.000 & 99 & 0.051 & 143 & 0.073 & 13 & 0.007 \\
\hline Paracyclops fimbriatus & 0 & 0.000 & 0 & 0.000 & 26 & 0.013 & 0 & 0.000 & 0 & 0.000 & 0 & 0.000 & 0 & 0.000 & 0 & 0.000 \\
\hline Afrocyclops gibsoni & 0 & 0.000 & 0 & 0.000 & 74 & 0.038 & 0 & 0.000 & 0 & 0.000 & 0 & 0.000 & 0 & 0.000 & 0 & 0.000 \\
\hline Eucyclops serrulatus & 0 & 0.000 & 0 & 0.000 & 0 & 0.000 & 0 & 0.000 & 0 & 0.000 & 51 & 0.026 & 0 & 0.000 & 0 & 0.000 \\
\hline Shizopera nilotica & 95 & 0.049 & 69 & 0.035 & 267 & 0.137 & 26 & 0.013 & 56 & 0.029 & 3704 & 1.899 & 61 & 0.031 & 0 & 0.000 \\
\hline Copepodite stage & 69 & 0.035 & 56 & 0.029 & 401 & 0.206 & 56 & 0.029 & 44 & 0.023 & 137 & 0.070 & 180 & 0.092 & 26 & 0.013 \\
\hline Nauplius stage & 0 & 0.000 & 0 & 0.000 & 0 & 0.000 & 26 & 0.013 & 56 & 0.029 & 0 & 0.000 & 0 & 0.000 & 0 & 0.000 \\
\hline Rotifera & 75 & $\begin{array}{c}0.038 \\
\end{array}$ & 93 & 0.048 & 82 & 0.042 & 123 & 0.063 & 26 & 0.013 & 1110 & 0.569 & 69 & 0.035 & 129 & 0.066 \\
\hline Cephalodella gibba & 24 & 0.012 & 61 & 0.031 & 0 & 0.000 & 105 & 0.054 & 26 & 0.013 & 382 & 0.196 & 13 & 0.007 & 39 & 0.020 \\
\hline Lecane lune & 51 & 0.026 & 32 & 0.016 & 0 & 0.000 & 18 & 0.009 & 0 & 0.000 & 395 & 0.203 & 0 & 0.000 & 51 & 0.026 \\
\hline Brachionus rubens & 0 & 0.000 & 0 & 0.000 & 0 & 0.000 & 0 & 0.000 & 0 & 0.000 & 333 & 0.171 & 0 & 0.000 & 26 & 0.013 \\
\hline Brachionus qudridentatus & 0 & 0.000 & 0 & 0.000 & 69 & 0.035 & 0 & 0.000 & 0 & 0.000 & 0 & 0.000 & 56 & 0.029 & 0 & 0.000 \\
\hline Brachionus zahniseri & 0 & 0.000 & 0 & 0.000 & 13 & 0.007 & 0 & 0.000 & 0 & 0.000 & 0 & 0.000 & 0 & 0.000 & 0 & 0.000 \\
\hline Brachionus sp. & 0 & 0.000 & 0 & 0.000 & 0 & 0.000 & 0 & 0.000 & 0 & 0.000 & 0 & 0.000 & 0 & 0.000 & 0 & 0.000 \\
\hline Brachionus urceolaris & 0 & 0.000 & 0 & 0.000 & 0 & 0.000 & 0 & 0.000 & 0 & 0.000 & 0 & 0.000 & 0 & 0.000 & 13 & 0.007 \\
\hline Trichocerca porcellus & 0 & 0.000 & 0 & 0.000 & 0 & 0.000 & 0 & 0.000 & 0 & 0.000 & 0 & 0.000 & 0 & 0.000 & 0 & 0.000 \\
\hline Ostracoda & 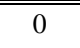 & 0.000 & 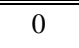 & 0.000 & 69 & 0.035 & 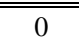 & 0.000 & 44 & 0.023 & 48 & 0.025 & 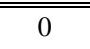 & $\begin{array}{c}0.000 \\
\end{array}$ & 93 & 0.048 \\
\hline Cypridopsis vidua & 0 & 0.000 & 0 & 0.000 & 56 & 0.029 & 0 & 0.000 & 44 & 0.023 & 48 & 0.025 & 0 & 0.000 & 93 & 0.048 \\
\hline Potamocypris variegate & 0 & 0.000 & 0 & 0.000 & 13 & 0.007 & 0 & 0.000 & 0 & 0.000 & 0 & 0.000 & 0 & 0.000 & 0 & 0.000 \\
\hline Candona sp. & 0 & 0.000 & 0 & 0.000 & 0 & 0.000 & 0 & 0.000 & 0 & 0.000 & 0 & 0.000 & 0 & 0.000 & 0 & 0.000 \\
\hline
\end{tabular}




\section{Dominancy of zooplankton taxa during the period of study:}

Using Engelmann's classification (Engelmann, 1978) in the treatment of zooplankton dominance structure, which reveals the classification: eudominant (40-100\%), dominant (12.5-39.9\%), subdominant (4-12.4\%), recedent (1.3-3.9\%), and subrecedent (bellow 1.3\%). Based on the heatmap of zooplankton species and dominancy were visually displayed among all samples. For instance, Chydorus sphaericus and Ceriodaphnia reticulata were the eudominant zooplankton taxa at all sites of the River Nile and canals. Mesocyclops ogunnus, Ilyocryptus sordidus, Bosmina longirostris, Thermocyclops consimilis, Thermodiaptomus galebi, Disparalona rostrata and Alona bukobensis (c) were the dominant zooplankton taxa Fig. (3 a,b).

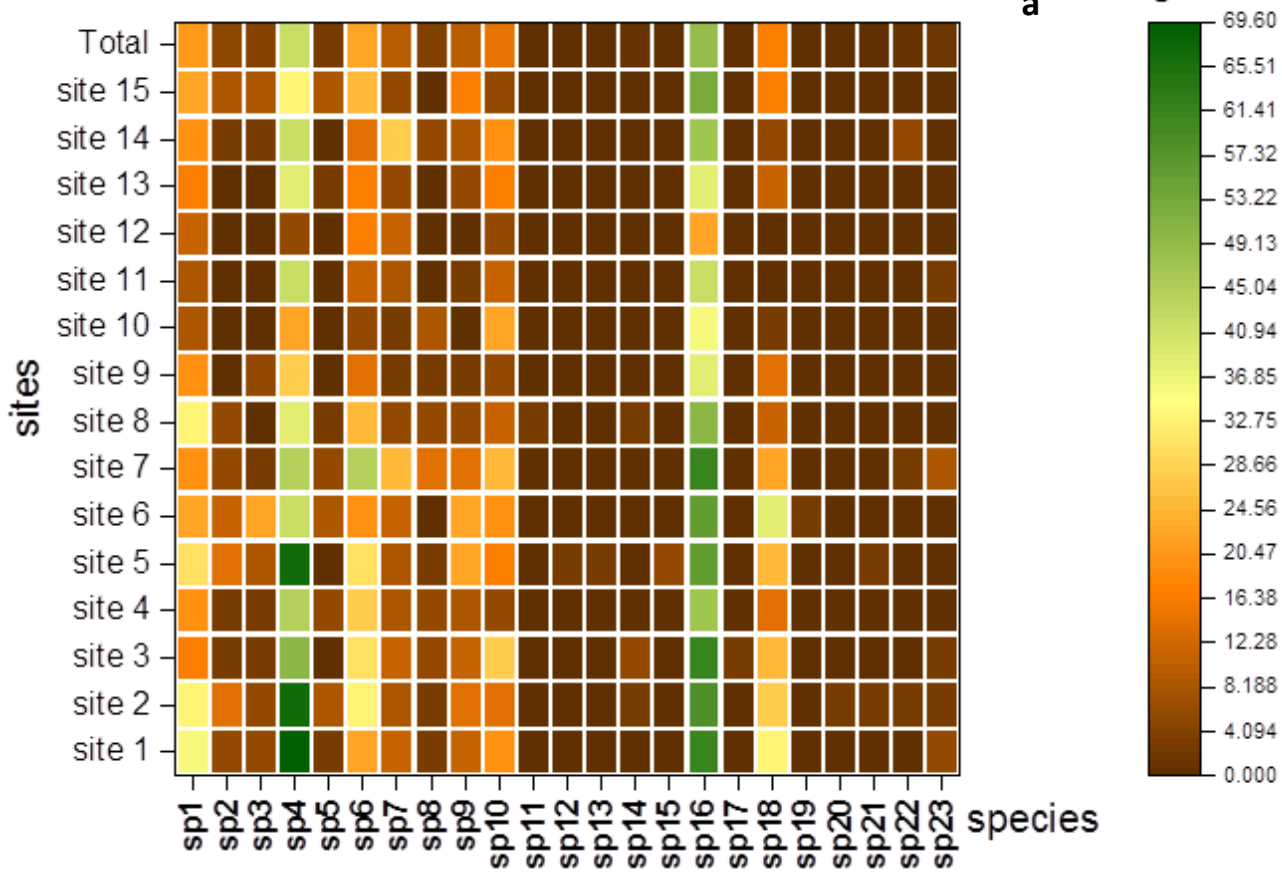

sp1- Bosmina longirostris, sp2- Simocephalus expinosus, sp3- Simocephalus vetulus, sp4- Ceriodaphnia reticulata, sp5- Daphnia longispina, sp6- Ilyocryptus sordidus, sp7- Macrothrix laticornis, sp8- Alona bukobensis (a), sp9- Alona bukobensis (b), sp10- Alona bukobensis (c), sp11- Alona rectangular, sp12Alona sp., sp13- Euryalona sp., sp14- Camptocercus australis, sp15-Leydigia quadrangularis, sp16Chydorus sphaericus, sp17- Chydorus sp., sp18- Disparalona rostrata,sp19- Pleuroxus straminius, sp20Pleuroxus aduncus, sp21- Dunhevedia crassa ,sp22-Diaphanosoma birgei, sp23- Moina micrura. 


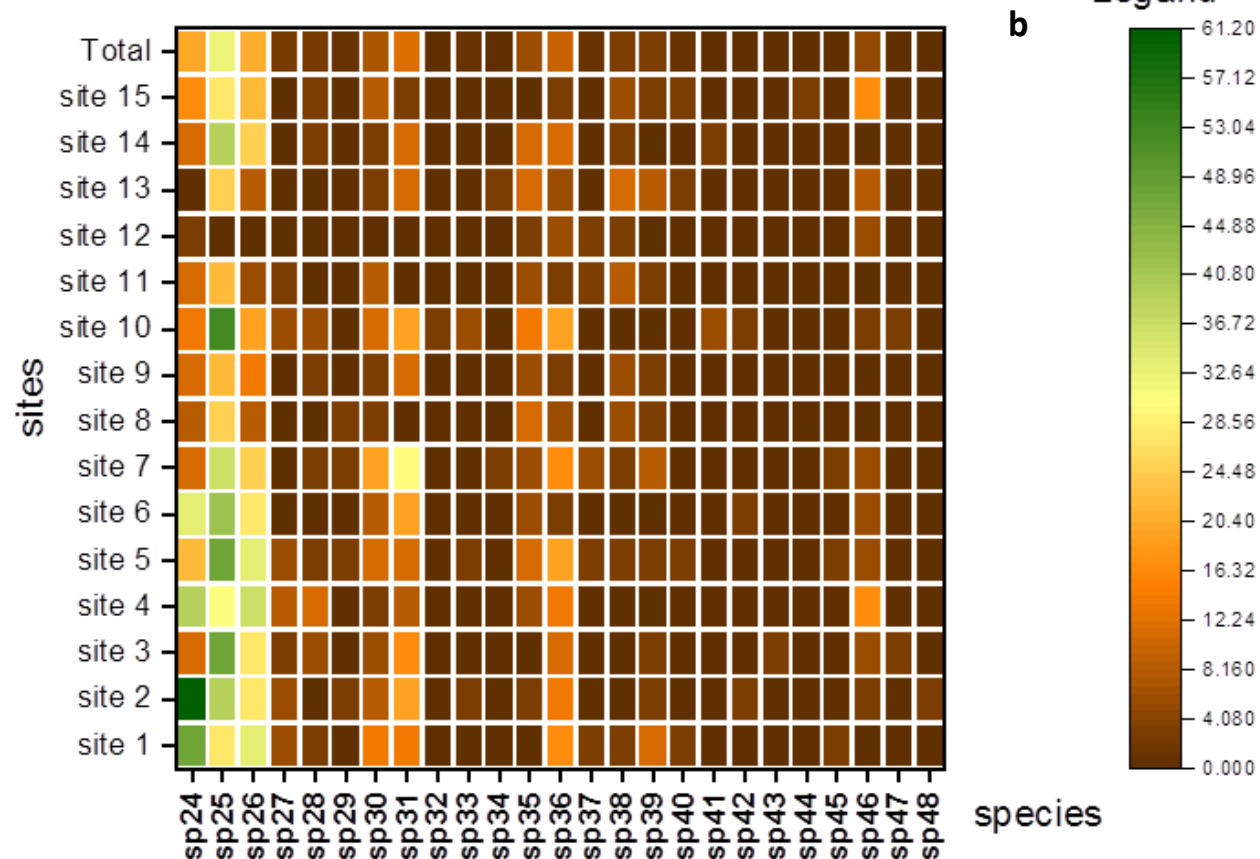

Fig. 3 (a,b). Heatmap of zooplankton species of the investigated sites (the data was based on the percentage of the dominance of taxa in all investigated site sites).

sp24-Thermodiaptomus galebi, sp25-Mesocyclops ogunnus, sp26-Thermocyclops consimilis, sp27Thermocyclops neglectus, sp28-Tropocyclops confinis, sp29-Macrocyclops albidus, sp30-Microcylops varicans, sp31-Microcylops linjanticus sp32-Paracyclops fimbriatus sp33-Afrocyclops gibsoni sp34Eucyclops serrulatus, sp35- Shizopera nilotica, sp36- Copepodite stage, sp37- Nauplius stage, sp38Cephalodella gibba, sp39- Lecane lune ,sp40-Brachionus rubens, sp41-Brachionus qudridentatus, sp42Brachionus zahniseri, sp43-Brachionus sp., sp44-Brachionus urceolaris, sp45- Trichocerca porcellus, sp46-Cypridopsis vidua, sp47- Potamocypris variegate, sp48- candona sp.

\section{Seasonal variations of water parameters:}

The hydrogen ion $(\mathrm{pH})$ showed the lowest value in spring and the highest value recorded in autumn. The seasonal mean value of total dissolved solids and conductivity recorded the lowest value in summer and the highest value recorded in winter. The seasonal mean value of turbidity recorded the lowest value in summer and the highest value recorded in autumn. The seasonal mean value of dissolved oxygen recorded the lowest value in summer and the highest value in autumn (Fig. 4). 


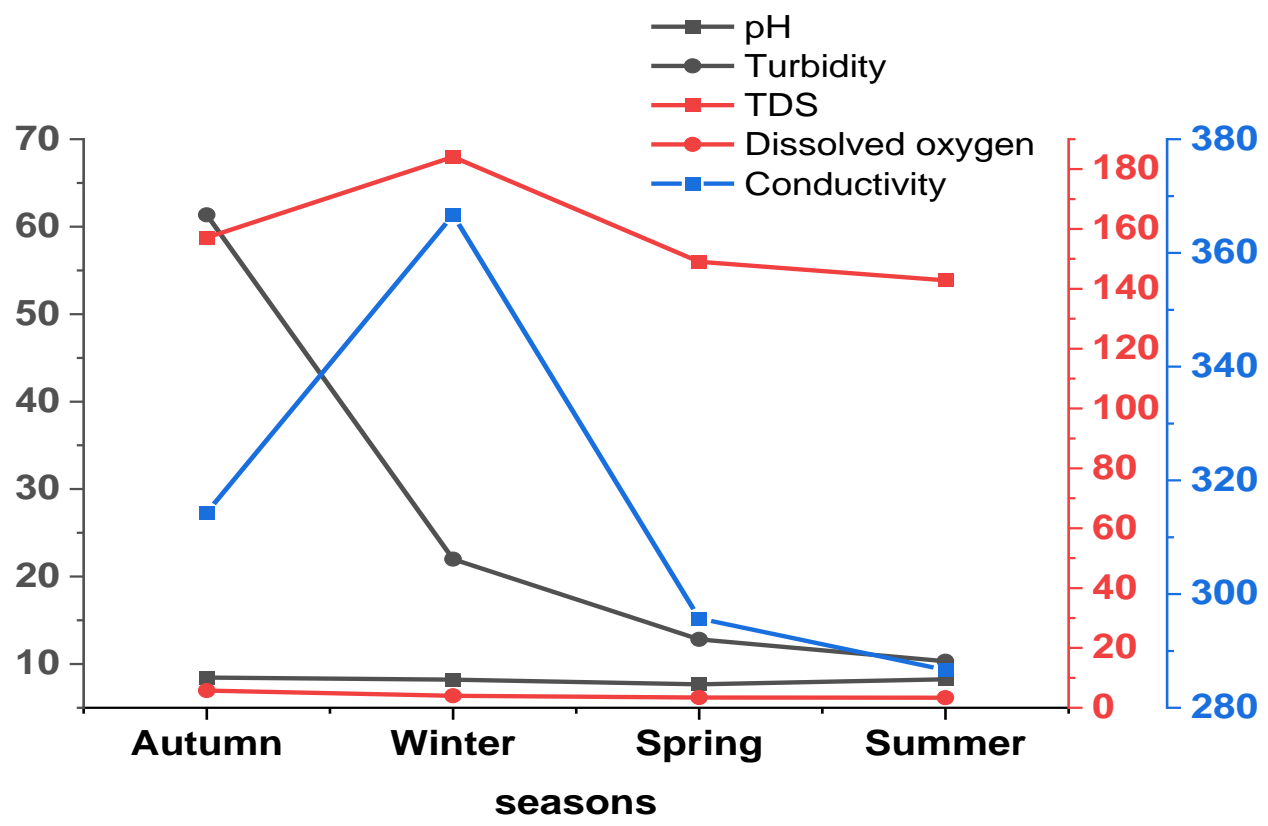

Fig. 4. Seasonal variations of water characters recorded during the period of investigation.

\section{Seasonal abundance of zooplankton taxa:}

The highest mean density of the total zooplankton taxa was recorded in winter and the lowest one was recorded in summer. Cladocera recorded high density during winter and low density during summer. The maximum density of Copepoda was observed during autumn, while the lowest was during summer. Rotifera recorded maximum density during winter and minimum was in summer. Ostracoda showed the highest density during autumn and spring, and no record of existence during summer (Table 3; Fig. 5).

Table 3. Seasonal mean density of zooplankton groups during the period of investigation

\begin{tabular}{cccccc}
\hline \hline Season & Cladocera & Copepoda & Rotifera & Ostracoda & Total zooplankton \\
\hline \hline Autumn & 772 & 246 & 8 & 4 & 1031 \\
Winter & 2002 & 215 & 29 & 2 & 2249 \\
Spring & 977 & 42 & 6 & 4 & 1028 \\
Summer & 20 & 6 & 1 & 0 & 27 \\
\hline \hline
\end{tabular}




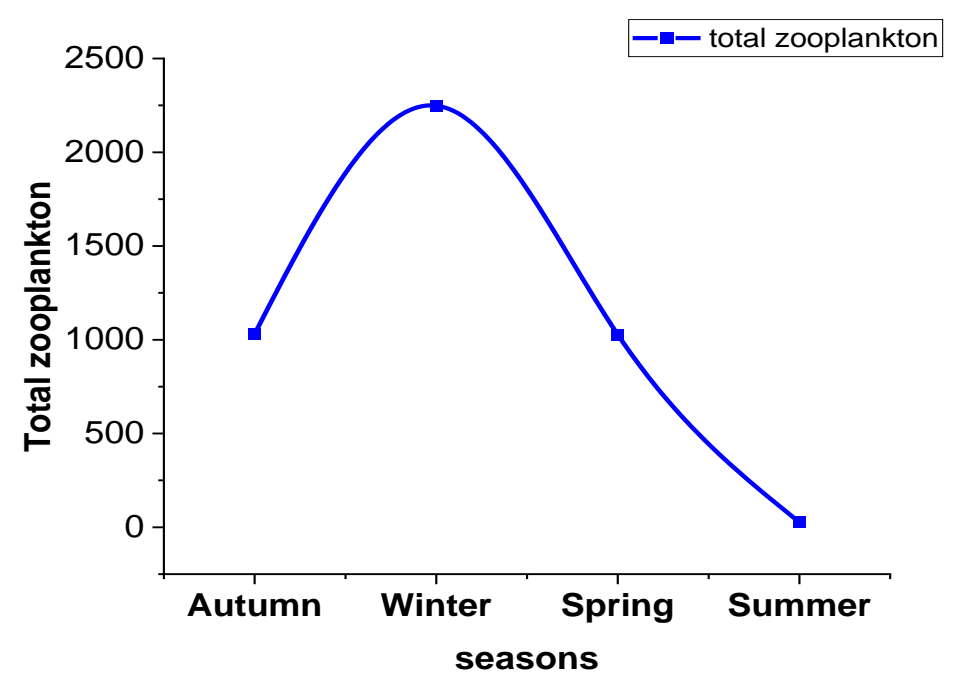

Fig. 5. Seasonal variations of total zooplankton during the period of investigation

The Canonical Correspondent Analysis (CCA) showed the relationship between environmental factors and zooplankton groups. Copepoda and Cladocera correlated positively with Turbidity and $\mathrm{pH}$. Site 6 recorded the highest value of turbidity. $\mathrm{pH}$ recorded the highest value during site 7 . Rotifera correlated positively with dissolved oxygen. Dissolved oxygen recorded the highest value in site 13. Ostracoda correlated positively with total dissolved solids and conductivity. Site 10 recorded the highest value of total dissolved solids and conductivity (Fig. 6).

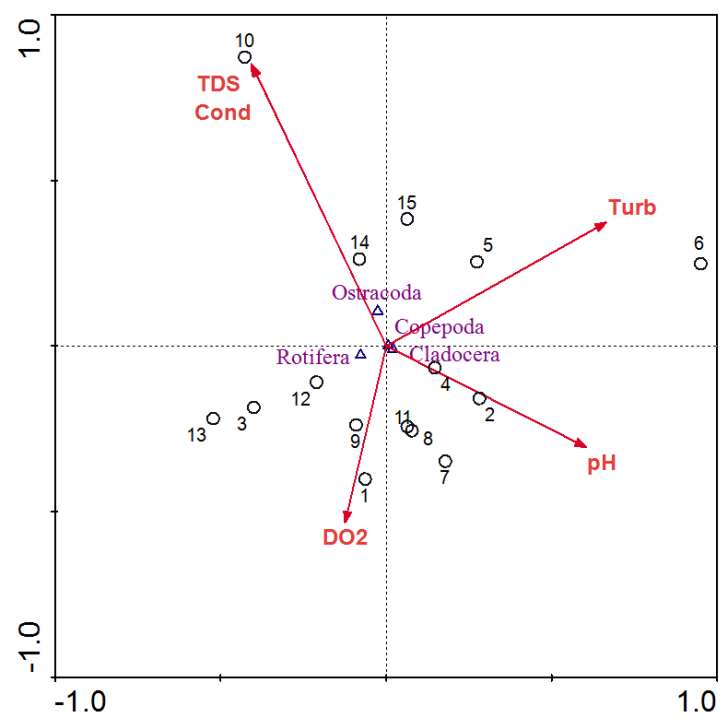

Fig. 6. CCA showing the correlation between zooplankton groups and environmental factors in different sites. 
Cluster analysis was used to study the similarity between the fifteen (15) studied sites in an abundance of zooplankton. The dendrogram showed that sites 1,2,5,6,3, and 15 formed separate cluster with similarity of $82 \%$. Also, sites 10,7 , and 14 formed a separate cluster with similarity of $66 \%$. Moreover sites 4,8,9,11, and 12 clustered together separately with $60 \%$, whilst Site 13 formed as an outlier with 0\% similarity Fig. (7).

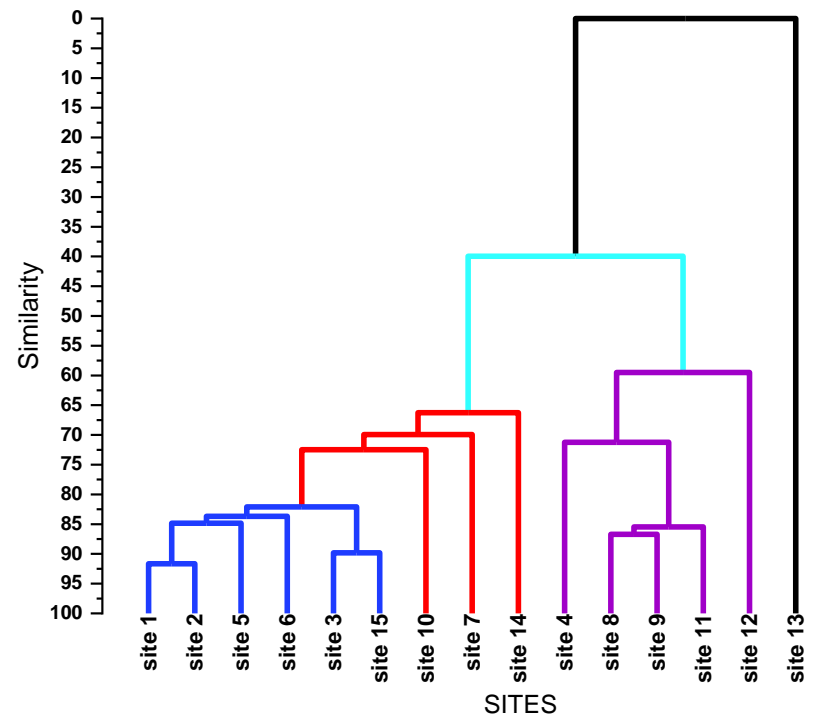

Fig. 7. Dendrogram for zooplankton abundance recorded in all investigated sites.

In the present study, the diversity indices fluctuated between sites through four seasons. The highest value of Taxa richness was recorded at site 7 during autumn (22 species), while no value of taxa richness was recorded at Sites 10,12 and 13 during summer. Shannon_H index (2.42) reached the highest value at site 9 during autumn, whilst no value was recorded at Sites 6,10,12 and 13 during summer. The highest Evenness value (1) was recorded at site 6 during summer and no value of Evenness was recorded at Site 10,12 and 13. Margalef index recorded the highest value (2.88) during autumn in site 3. Equitability_J showed the highest value (0.91) at site 12 during autumn. There is no value was recorded during summer at site 6,10,12 and 13 for both indices Margalef and Equitability_J (Fig.8). 
PCA axes 1 and 2 represent $97.54 \%$ of the total variation in zooplankton diversity. Pc 1 representing the major axis of variation with a (82.89\%) variance explained positive relation in sites 12,8,4,9,13,11 with Shannon_H, Evenness and Equitability_J. Moreover site 3 related negatively with these variables. Pc 2 representing a (14.65\%) variance explained Taxa_S and Margalef related positively with sites 5,2,7,1 and related negatively with site 12 Fig. (9).

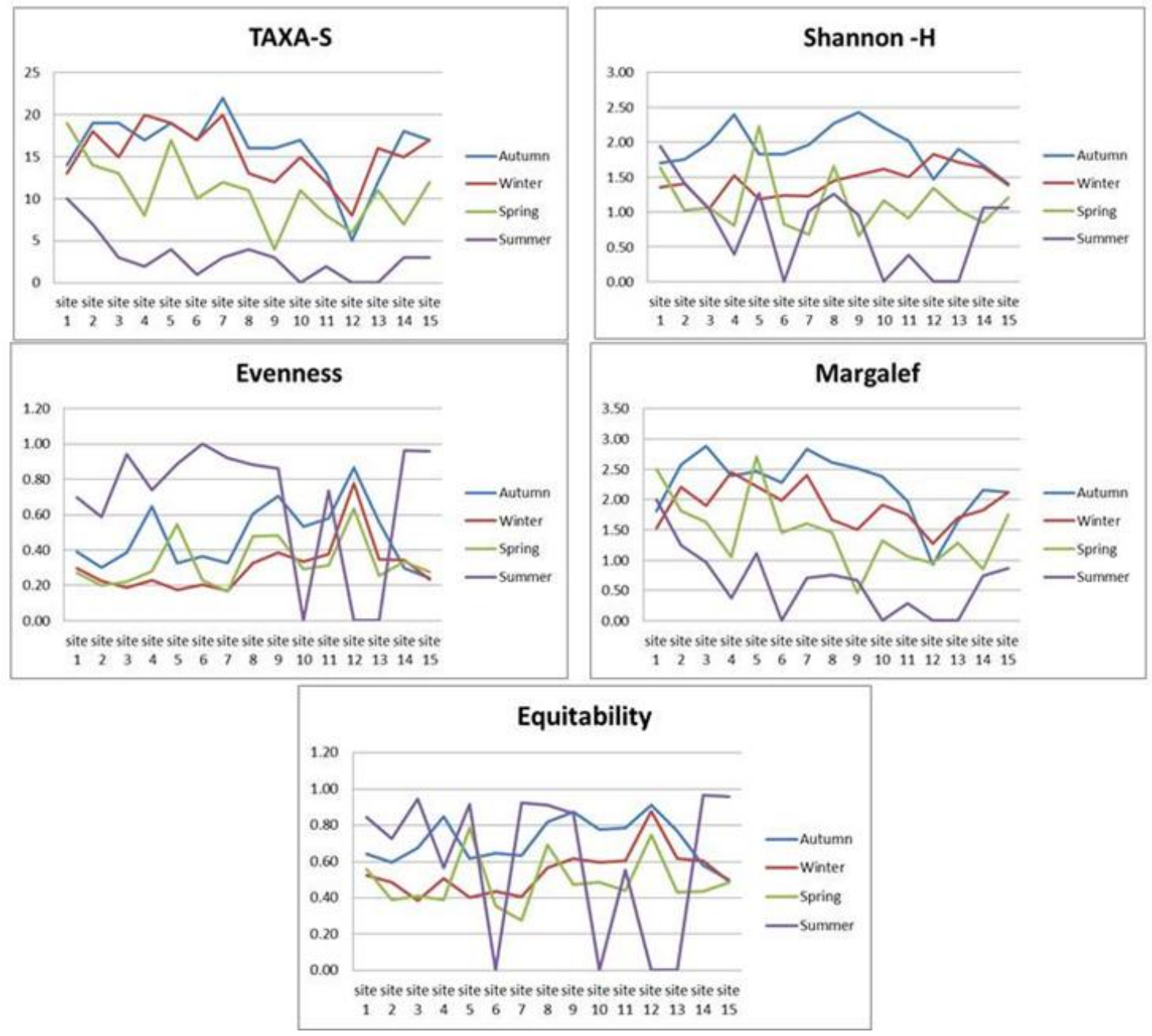

Fig. 8. Seasonal variation in diversity indices of zooplankton distribution during the period of investigation in all sites. 


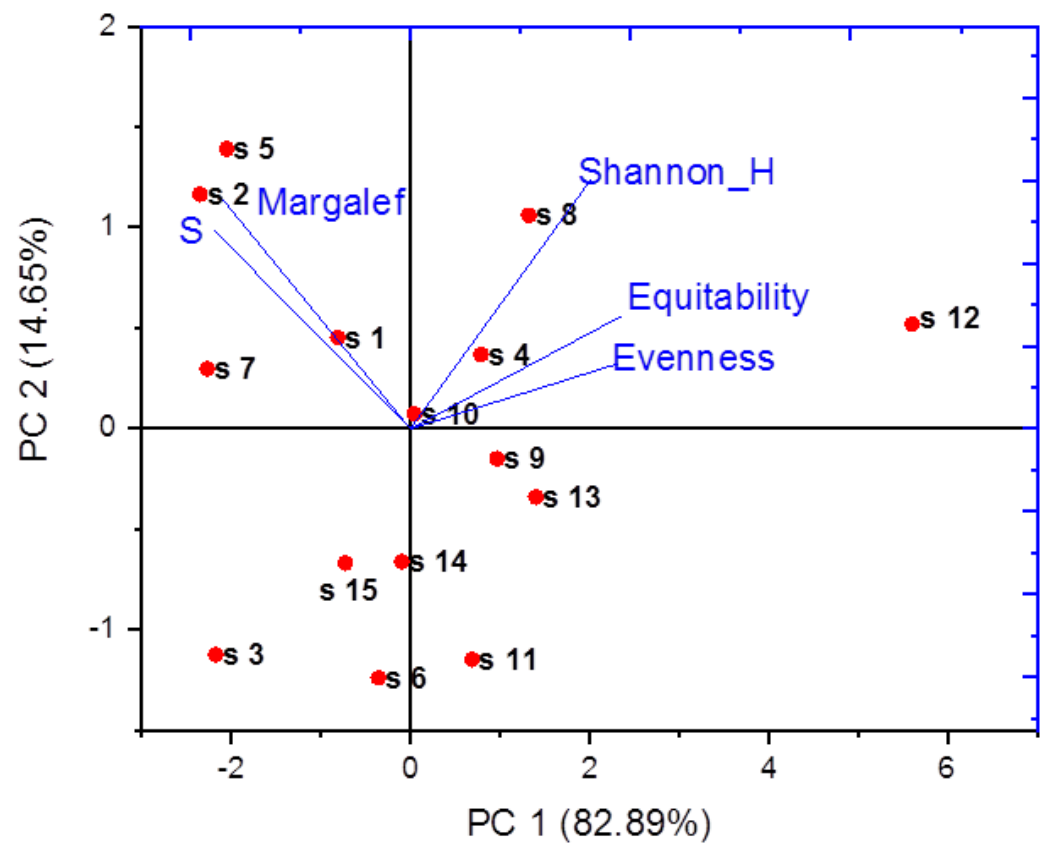

Fig. 9. PCA ordination graph showing the diversity indices of zooplankton distribution during the period of investigation in all investigated sites. 1=site $12=$ site $2 \quad 3=$ site 3 $4=$ site $4 \quad 5=$ site $5 \quad 6=$ site $6 \quad 7=$ site 7 =site $8 \quad 9=$ site $9 \quad 10=$ site10 $11=$ site $11 \quad 12=$ site 12 $13=$ site $1314=$ site $1415=$ site 15 .

\section{DISCUSSION}

Zooplankton represent the major components of aquatic invertebrate fauna. Therefore, any changes in the zooplankton population dynamics, community structure and function reflect water quality and its developmental trend (Yang et al., 2017; Berraho et al., 2019). In freshwater ecosystems, planktonic groups at a certain moment are composed of one dominant species and others in a large or small fraction of the total population (Pennak, 1957).

In the present study, the total density of zooplankton was higher in winter and less in summer. This result may be attributed to favorable environmental conditions such as temperature, dissolved oxygen and the availability of rich nutrients in the form of bacteria, nano-plankton and suspended detritus. Low water temperature is adverse for predation while high dissolved oxygen could adversely flourish the growth of zooplankton. San et al. (2006) indicated that over zooplankton growth increases with relatively low temperatures. Randive et al. (2015) stated that dissolved oxygen is necessary for aquatic species to regulate metabolic processes and for animal respiration. According to the data, the abundance of zooplankton species was high in site 13 and the 
lowest in site 12 . Increasing the density in site 13 could be related to the elevation of dissolved oxygen in the water.

Cladocera was the most dominant group, followed by Copepoda. The highest population density of Cladocera was observed during the winter season, and the lowest population density was in the summer. This result agreed with Obuid-Allah, et al. (2019, 2020). Cladoceran populations peak during winter indicating that they may be linked to a combination of suitable temperature and abundance of food in the form of bacteria and suspended detritus. Flourishing of Cladocera in the cold weather is in accordance with Green (1962) who concluded that most large cladoceran species don't reproduce in summer but can in winter and spring. Bedair (2006) concluded that the decrease in Cladocera population density during summer may be attributed to the abundance of bluegreen algae and dinoflagellates, which impede the Cladocera filtering rate. Furthermore, Pandey et al. (2009) indicated that the decreased abundance of Cladocera throughout summer can be linked to fish predation and active competition with other groups.

In the present investigation, Chydorus sphaericus was the most dominant species, followed by Ceriodaphnia reticulata then Mesocyclops ogunnus. The dominancy of Chydorus sphaericus may be related to its high ability to adapt to all environmental changes. Basińska (2014) suggested that $C$. sphaericus is a cladoceran with a wide range of distribution and can be found in a variety of aquatic habitats because it might be able to adapt to changes in oxygen levels. Ceriodaphnia reticulata was the second dominant species, this result is in concurred with Mahmoud (1995) who stated that Ceriodaphnia reticulata was a common species, accounting for $23.5 \%$ of the overall population.

In aquatic ecosystems, water is influenced by various environmental factors including physical properties (gases and solids solubility, light penetration, temperature and density) and chemical properties (salinity, $\mathrm{pH}$, hardness, phosphates and nitrates). These factors are very important for the growth and dispersal of phytoplankton and zooplankton.

The present study, observed variations in physicochemical parameters of water, species composition, population density, species diversity, species evenness and species richness of different zooplankton. The $\mathrm{pH}$ is the scale that measures the concentration of $\mathrm{H}+$ ions and measures the intensity of acidity and alkalinity of water. The highest value of $\mathrm{pH}$ was recorded during autumn, this result agreed with Nwinyimagu et al. (2021) who detected the highest mean value in the wet season. The study showed that Claodcera correlated positively with $\mathrm{pH}$. This result agrees with Karuthapandi et al. (2012) and deepthi et al. (2014) who observed a positive correlation between Cladocera and alkalinity. Nevalainen et al. (2013); \& Zawiska et al. (2013) indicated that Cladocera species are particularly sensitive to $\mathrm{pH}$ fluctuations and this reflects their sensitivity to acidification. Belyaeva \& Deneke (2007); Zawisza \& Cedro (2012) concluded that 
Chydorus sphaericus is the most widespread cladoceran taxon across Europe, reflecting its wide ecological tolerance, especially to $\mathrm{pH}$.

The seasonal mean value of total dissolved solids and conductivity was highest in winter, this conclusion matched with Obuid-Allah, et al. (2020). The highest mean value of total dissolved solids might be attributed to the accumulation of the anthropogenic activity, which hampered the quality of water (Ezhili, et al., 2013; Manickam et al., 2014, 2015; Bhavan et al., 2015). The increase in suspended solids in the water ecosystem may cause the rapid growth of algae, which is a very significant food source for many zooplanktons. The highest average value of turbidity was recorded in autumn, this conclusion is consistent with Obuid-Allah, et al. (2020). Welcomme (1979) found substantial turbidity during the winter months. This may be attributed to high quantities of total dissolved compounds in the water brought by rains and soil drift. The seasonal mean value of dissolved oxygen showed the least during summer, this result is consistent with Saravanakumar et al. (2021).

In both freshwater and marine water, plankton diversity was the most significant ecological parameter. The Shannon-Wiener diversity index is directly connected to the number, uniform distribution, and total abundance of species in a sample (Benedict et al., 2011). Species richness refers to different types of species and their numerical strength also, it refers to the ratio between different species $(\mathrm{S})$ and a total number of species $(\mathrm{N})$. Species evenness is a measure that qualifies how even species are in terms of their number. The higher value of Shannon's index $\left(\mathrm{H}^{\prime}\right)$ and the population of zooplankton were recorded during summer while the lower values were recorded during monsoon months.

In the present study, a higher value of Taxa richness (S) was recorded during autumn also, a high value of Shannon's index $\left(\mathrm{H}^{\prime}\right)$ was recorded during autumn. This result is in accordance with Manickam et al. (2018) who found that the species richness of zooplankton was higher in post-monsoon to monsoon whereas, the lower value was in summer and pre-monsoon season. The high zooplankton diversity may be related to large food availability and suitable environmental conditions for the growth and development of their populations. Manickam et al. (2015) mentioned that the high species diversity of zooplankton in the perennial lake indicates the least pollution. The species equitability (evenness) was relatively high during the rainy season indicating a reduction in the plankton diversity at this period (Adesalu \& Nwankwo, 2008).

Furthermore, high value of Marglef index was recorded during autumn, whilst the high value of Evenness was observed during summer. The same result for each the Marglef and Evenness indices was noticed in Au River, Nigeria by Nwinyimagu et al. (2018). The high value of species equitability was observed during autumn, this is 
concurrs with Adesalu \& Nwankwo (2008) who noticed a relatively high value of equitability during the rainy season.

\section{CONCLUSION}

In conclusion, there were significant seasonal variations in zooplankton community in the 15 investigated sites, with cladocerans dominating, followed by copepods. The numerical density, Taxa richness, Shannon diversity index, Marglef index and Equitability of zooplankton were higher in the Rainy season, with a decrease in the dry season. The environmental conditions were one of the most important factors controlling the seasonal variation in the size of the zooplankton community.

\section{REFERENCES}

Adesalu, T. A. and Nwankwo, D. I. (2008). Effect of water quality indices on phytoplankton of a sluggish tidal creek in Lagos, Nigeria. Pak. J. boil. Sci. PJBS, 11(6): 836-844.

Allan, J. D. (1975). The distributional ecology and diversity of benthic insects in Cement Creek, Colorado. Ecology. 56(5):1040-53.

Basińska, A. M. ; Antczak, M.; Świdnicki, K.; Jassey, V. E. J.; and KuczyńskaKippen, N. (2014). Habitat type as strongest predictor of the body sizedistribution of Chydorus sphaericus (O. F. Müller) insmall water bodies. Int. Rev. Hydrobiol. 99: 382-392.

Bedair, S. M. (2006). Environmental studies on zooplankton and phytoplankton in some polluted areas of the River Nile and their relation with feeding habit of fish. $\mathrm{Ph}$. D. Thesis, Fac. Of Sci. Zagazig Uni.

Belyaeva, M. and Deneke, E. R. (2007). Colonization of acidic mining lakes: Chydorus sphaericus and other Cladocera within a dynamic horizontal $\mathrm{pH}$ gradient $(\mathrm{pH} 3.7)$ in Lake Senftenberger See (Germany). Hydrobiol. 594:97-108.

Benedict, O. O.; Ezekiel, O. A.; Gabriel, U. I.; Ada, F. B. and Steven, N. O. (2011). Plankton-based assessment of the trophic state of three tropical lakes. J. Env. Prot 2:304-315

Berraho, A. ; Abdelouahab, H. ;Baibai, T. ;Charib, S. ;Larissi, J. ; Agouzouk, A. and Makaoui, A. (2019). Short-term variation of zooplankton community in Cintra Bay (Northwest Africa). Oceanolog, 61(3): 368-383. 
Bhavan, P. S.; Selvi, A.; Manickam, N.; Srinivasan, V.; Santhanam, P.; and Vijayan, P. (2015). Diversity of zooplankton in a perennial Lake at Sulur, Coimbatore, India. Int. J. Int. J. Ext. Res, 5: 31-44.

Brooks, J. L. (1959). Cladocera In : Fresh water biology, 2nd ed. Edmondson W. T.

Deepthi, S. and Yamakanamardi, S. M. (2014). Abundance of cladoceran zooplankton in varuna, madappa and giribettethe lakes of mysore, Karnataka state, india. Int. J. Sci. Env. 3(3):885 - 900.

Egborge, A. B. M. (1994). Water pollution in Nigeria: Biodiversity and chemistry of Warri River. Ben-Miller Books Nigeria Ltd, pp.27-59.

Elfeky, F. A., and Sayed, N. K. (2014). Distribution and abundance of rotifers in the River Nile, Egypt. Wor. J. Fis. Mar. Sci., 6 (6): 557-563.

Ezhili, N. Manikandan, R. and Ilangovan, R. (2013). Diversity and seasonal variation of zooplankton in Ukkadam Lake, Coimbatore, Tamil Nadu, India. Int. J. Cur. Res. 5(8): 2091-2094.

González, C. E.; Escribano, R., Bode, A. and Schneider, W. (2018). Zooplankton taxonomic and trophic community structure across biogeochemical regions in the eastern South Pacific. Front Mar Sci 5: 498pp.

Green, J. (1962). Zooplankton of the River Socoto. The Rotifera: The crustacea. Proc. Zod. Soc. London, 138(4): 414-453.

Güher, H. (2014). A checklist for zooplankton (Rotifera, Copepoda, Cladocera) of European Turkey inland waters. Su Ürünleri Dergisi 31: 221-225.

Gupta, M.; Kumar, P; Mishra, U.K. and Kumar, D. (2016). Planktonic diversity and density in Keerat Sugar pond at Mahoba District of Ultar Pradesh, India.International Journal of Innovative Research and Development, 5(7):181186.

Karuthapandi, M.; Xavier Innocent, B., and Siddiqi, S. Z. (2012). Zooplankton in a temporary freshwater pond habitat in Attapur, Hyderabad, Andhra Pradesh, India. Int. J. Adv. Li. Sci. (IJALS), 1: 22-31.

Lin, Q. Q.; Duan, S. S.; Hu, R., and Han, B. P. (2003). Zooplankton distribution in tropical reservoirs, South China. Int. Rev. Hydrobiol: A Journal Covering all Aspects of Limnology and Marine Biology, 88(6): 602-613.

Madhusudhana, R. k.; Krishna, P. V.; Jyothirmayi, V. and Hemanth, K. V. (2014). Biodiversity of zooplankton communities in a Perennial pond at lake Kolleru region of Andhra Pradesh, India. Int. J. Adv. Res., 2(7):33-41. 
Mahmoud, A. A. (1995). Taxonomical and ecological studies on freshwater fleas (Cladocera) in Qena governate. Ph.D.Thesis Zool.Depart.Fac.Sci.Qena,south valley Univ.Egypt.

Manickam, N. ; Bhavan, P. S.; Santhanam, P. ; Bhuvaneswari, R. ; Muralisankar, T. ; Srinivasan, V. and Karthik, M. (2018). Impact of seasonal changes in zooplankton biodiversity in Ukkadam Lake, Coimbatore, Tamil Nadu, India, and potential future implications of climate change. J. Basic and App. Zoo., 79(1): 110.

Manickam, N.; Bhavan, P. S.; Santhanam, P.; Muralisankar, T.; Srinivasan, V.; Vijayadevan, K., and Bhuvaneswari, R. (2015). Biodiversity of freshwater zooplankton and physico-chemical parameters of Barur Lake, Krishnagiri District, Tamil Nadu, India. Mal. J. Biosci., 2: 1-12.

Manickam, N.; Bhavan, P. S.; Santhanam, P.; Muralisankar, T.; Srinivasan, V.; and Radhakrishnan, S. (2014). Seasonal variations of zooplankton diversity in a perennial reservoir at Thoppaiyar, Dharmapuri District, South India. J. Aqua \& Mar. Biol., 1(1): 1-7.

Margalef, R. (1968). Perspectives in Ecological Theory. Univ. of Chicago Press,

Nevalainen, L.; Luoto, T. P; Kultti, S. and Sarmaja-Korjonen, K. (2013). Spatiotemporal distribution of sedimentary Cladocera (Crustacea: Branchiopoda) in relation to climate. J. Biog. 40:1548-1559. New York, pp 587-656. John Wiley and sons.

Nwinyimagu, A. J.; Eyo, J. E. and Okogwu, O. I. (2021). Seasonal variation in abundance and diversity of zooplankton in Asu River، Ebonyi state, Nigeria. Acta Ecologica Sinica, In press.

Nwinyimagu, J. A.; Idumah, O. O. and Godwin, N. (2018). Seasonal Variation in Physicochemical Parameters and its Relationship with Zooplankton Abundance in River Asu, Nigeria. Ind. J. Eco., 45(1): 60-65.

Obuid-Allah, A. H. (2001). A review article on: Diversity of Cladocera and Copepoda in the freshwater bodies of Egypt and factors affecting it. Zool. Depart. Fac. Sci., Assiut Univ.

Obuid-Allah, A. H. ; Moustafa, A .S. ; Hussien, EL. H. M. ; Gaber ,A. M. and Mohammad, W. A. (2020). Distribution of Zooplankton Community in the River Nile at Esna Barrages,Egypt in relation to some ecological factors. Egyp. J. Aqu. Biol. \& Fish. 24(1): 131 - 143. 
Obuid-Allah, A. H. ; Moustafa, A. S.; Mohammad, W.A.; Hussien, EL. H. M. and Gaber, A. M. (2019). Community structure of crustacean Zooplankton in the River Nile at Esna Barrages, Upper Egypt. Egyp. J. Aqu. Biol. \& Fish. 23(4): 685 -695 .

Okechukwu, I. O. (2019). Seasonal variations of species composition and abundance of zooplankton in Ehoma Lake, a floodplain lake in Nigeria. Int. J. Trop. Biol. 58 (1): 171-182.

Pandey, B. N.; Ambasta, O. P.; Thakur, A. K.; Kumar, S. and Kumari, R. (2009). Zooplankton diversity in relation to certain physic-chemical parameters of swamp of Kishanganj, Bihar. Enviro. Conserv. J., 10 (1 \& 2): 9 - 14.

Pennak, R. W. (1957). Species Composition of Limnetic Zooplankton Communities 1. Limn. and Oceanog. 2(3): 222-232.

Pielou, E.C. (1975). Ecological diversity. John Wiley and Sons, New York.

Randive, S. R. and Kamble, V. S. (2015). Studies On Some Physico-Chemical Parameters With Reference To Zooplankton Of Walekhindi Tank From Jath Taluka Dist-Sangali. Int. J. Adv. App. Res. (IJAAR).2(4):87-93.

Rita, P.; Gilia, P; Rómulo, R; Carlos, L. (2005). Limnological studies on a shallow reservoir in western Venezuela (Tulé Reservoir). 31(2):139-145.

San, M., E.; Harris, R. P.; and Irigoien, X. (2006). Latitudinal variation in plankton size spectra in the Atlantic Ocean. Deep Sea Research Part II: Topical Studies in Oceanography. 53(14-16):1560-1572.

Saravanakumar, M.; Murugesan, P.; Damotharan, P., and Punniyamoorthy, R. (2021). Seasonal Composition and Diversity of Zooplankton in Pichavaram Mangrove Forest, Southeast Coast of India. Int. J. Mod. Tre. Sci. Tech., 7(09): 6070.

Savitha, N. and Yamakanamardi, S. M. (2012). Studies on abundance of zooplanktons in lakes of Mysore, India. J Environ Biol.33(6):1079-85.

Shannon CE, Weaver, W. (1963). The mathematical theory of communications.

ter Braak, C. J. F., Smilauer, P. (2002). CANOCO reference manual and CanoDraw for Windows user's guide: Software for Canonical Community Ordination (version 4.5). Biometris, Wageningen.

Trivedi, S.; Siddiquw, A. A. and Rawat, R. (2015). Studies of zooplankton ecology in TriveniSangam, Kshipra River Ujjain (M.P). Int. J. Pharmacol. Bio. Sci., 9(3):4954. 
Varadharajan, D.; Soundarapandian, P.; Dinakaran ,G. K. and Vijaykumar, G. (2009). Crab Fishery Resources from Arukkattuthurai to Aiyammpattinam, South East Coast of India. Curr Res J Biol Sci.1: 118-122.

Welcomme, R. L. (1979). Fisheris Ecology of Flood Plain Rivers. Longman International, New York (317 pp.).

Wilhm, J. L and Dorris, T. C. (1968). Biological parameters for water quality criteria. Bioscience. 1:477-481.

Wilson, M. S. and Yeatman, H. C. (1959). Free living copepod In: ward, H. B. and Whpple, C. C. Freshwater Biology, 2nd ed. 1959 (ed. W. T. Edondson), pp. 735868.

Yang, J.; Zhang, X.; Xie, Y.; Song, C.; Zhang, Y.; Yu, H. and Burton, G. A. (2017). Zooplankton community profiling in a eutrophic freshwater ecosystem-lake tai basin by DNA metabarcoding. Scientific Reports. 7(1): 1-11.

Zawiska, I. Zawisza, E. Woszczyk, M. Szeroczyńska, K. Spychalski, W. CorreaMetrio, A. (2013). Cladocera and geochemical evidence from sediment cores show trophic changes in polish dystrophic lakes. Hydrobiol. 715:181-193.

Zawisza, E. and Cedro, B. (2012). Subfossil cladocera (Crustacea) fauna from sediments of Samowo drainage paleochannels (core T28). In: Cedro B (ed). 